\title{
Reasoning about Epistemic States of Agents by Modal Logic Programming
}

\author{
Linh Anh Nguyen \\ Institute of Informatics, University of Warsaw \\ ul. Banacha 2, 02-097 Warsaw, Poland \\ nguyen@mimuw.edu.pl
}

\begin{abstract}
Modal logic programming is one of appropriate approaches to deal with reasoning about epistemic states of agents. We specify here the least model semantics, the fixpoint semantics, and an SLD-resolution calculus for modal logic programs in the multimodal logic $K D 4 I_{g} 5_{a}$, which is intended for reasoning about belief and common belief of agents. We prove that the presented SLD-resolution calculus is sound and complete. We also present a formalization of the wise men puzzle using a modal logic program in $K D 4 I_{g} 5_{a}$. This shows that it is worth to study modal logic programming for multi-agent systems.
\end{abstract}

\section{Introduction}

Reasoning is an important aspect of agents. In order to be able to make right actions, an agent should have general knowledge of the field it works on, information about the environment, and abilities to interact with the environment, to make inferences, and to revise its knowledge base. In multi-agent systems, agents should be able to communicate, collaborate, and sometimes compete with each other. For this aim, an agent should have knowledge about other agents in the system and be able to reason about their epistemic states. It is not that an agent can have all information it wants or can reason exactly as the others, but at least it can simulate epistemic states of the other agents, using some assumptions. The wise men puzzle introduced by McCarthy [17] is an example of reasoning about epistemic states of agents. We will study it in Section 3.

Modal logics and logic programming are useful instruments for multi-agent systems. Using modal logics is a natural way to represent and reason about knowledge and belief of agents (see, e.g., $[10,28,27,14,7,1]$ ). Logic programming is also useful because logical implication is probably the inference form humans use most and want to adopt for multi-agent systems. Thus, one can think about modal logic programming as an approach to deal with reasoning about epistemic states of agents.

Modal logic programming has been studied in a number of works (see the earlier surveys $[24,12]$ and the later works $[23,5,19,22])$. There are two approaches: the direct approach $[11,3,5,19,22]$ and the translation approach $[8$, $23]$. The first approach directly uses modalities, while the second one translates 
modal logic programs to classical logic programs. In this paper we will use the direct approach. This approach is justifiable, as the direct approach deals with modalities more closely, and modalities allow us to separate object-level and epistemic-level notions nicely.

In [19], we developed a fixpoint semantics, the least model semantics, and an SLD-resolution calculus in a direct way for modal logic programs in all of the basic serial monomodal logics. In that work we do not assume any special restriction on occurrences of $\square$ and $\diamond$ in programs and goals. In [22], we generalized the methods of [19] and gave a general framework for developing fixpoint semantics, the least model semantics, and SLD-resolution calculi for logic programs in normal multimodal logics whose frame restrictions consist of the conditions of seriality and some classical first-order Horn formulas.

In this work, we instantiate the above mentioned framework for the multimodal logic $K D 4 I_{g} 5_{a}$, which was introduced in [20] for reasoning about belief and common belief. We prove that the obtained SLD-resolution calculus is sound and complete. We also give a purely logical formalization of the wise men puzzle using a modal logic program in $K D 4 I_{g} 5_{a}$.

The rest of this paper is structured as follows. In Section 2, we give definitions for multimodal logics, define the multimodal logic $K D 4 I_{g} 5_{a}$ and the modal logic programming language MProlog. In Section 3, we recall the wise men puzzle and formalize it by an MProlog program in $K D 4 I_{g} 5_{a}$. In Section 4, we instantiate the framework given in [22] for $K D 4 I_{g} 5_{a}$ in order to specify the least model semantics, the fixpoint semantics, and an SLD-resolution calculus for MProlog programs in $K D 4 I_{g} 5_{a}$. Soundness and completeness of the obtained SLD-resolution calculus is proved in Section 5. (Due to the lack of space we do not present proofs involving with the fixpoint semantics and the least model semantics.) Finally, Section 6 contains some concluding remarks.

\section{Preliminaries}

\subsection{Syntax and Semantics of Quantified Multimodal Logics}

A language for quantified multimodal logics is an extension of the language of classical predicate logic with modal operators $\square_{i}$ and $\diamond_{i}$, for $1 \leq i \leq m$ (where $m$ is fixed). The modal operators $\square_{i}$ and $\diamond_{i}$ can take various meanings. For example, $\square_{i}$ can stand for "the agent $i$ believes" and $\diamond_{i}$ for "it is considered possible by agent $i$ ". The operators $\square_{i}$ are called universal modal operators, while $\diamond_{i}$ are called existential modal operators. Terms and formulas are defined in the usual way, with an emphasis that if $\varphi$ is a formula then $\square_{i} \varphi$ and $\diamond_{i} \varphi$ are also formulas.

A Kripke frame is a tuple $\left\langle W, \tau, R_{1}, \ldots, R_{m}\right\rangle$, where $W$ is a nonempty set of possible worlds, $\tau \in W$ is the actual world, and $R_{i}$ is a binary relation on $W$, called the accessibility relation for the modal operators $\square_{i}, \diamond_{i}$. If $R_{i}(w, u)$ holds then we say that the world $u$ is accessible from the world $w$ via $R_{i}$.

A fixed-domain Kripke model with rigid terms, hereafter simply called a Kripke model or just a model, is a tuple $M=\left\langle D, W, \tau, R_{1}, \ldots, R_{m}, \pi\right\rangle$, where 
$D$ is a set called the domain, $\left\langle W, \tau, R_{1}, \ldots, R_{m}\right\rangle$ is a Kripke frame, and $\pi$ is an interpretation of constant symbols, function symbols and predicate symbols. For a constant symbol $a, \pi(a)$ is an element of $D$, denoted by $a^{M}$. For an $n$-ary function symbol $f, \pi(f)$ is a function from $D^{n}$ to $D$, denoted by $f^{M}$. For an $n$-ary predicate symbol $p$ and a world $w \in W, \pi(w)(p)$ is an $n$-ary relation on $D$, denoted by $p^{M, w}$.

A model graph is a tuple $\left\langle W, \tau, R_{1}, \ldots, R_{m}, H\right\rangle$, where $\left\langle W, \tau, R_{1}, \ldots, R_{m}\right\rangle$ is a Kripke frame and $H$ is a function that maps each world of $W$ to a set of formulas.

Every model graph $\left\langle W, \tau, R_{1}, \ldots, R_{m}, H\right\rangle$ corresponds to a Herbrand model $M=\left\langle\mathcal{U}, W, \tau, R_{1}, \ldots, R_{m}, \pi\right\rangle$ specified by: $\mathcal{U}$ is the Herbrand universe (i.e. the set of all ground terms), $c^{M}=c, f^{M}\left(t_{1}, \ldots, t_{n}\right)=f\left(t_{1}, \ldots, t_{n}\right)$, and $\left(\left(t_{1}, \ldots, t_{n}\right) \in\right.$ $\left.p^{M, w}\right) \equiv\left(p\left(t_{1}, \ldots, t_{n}\right) \in H(w)\right)$, where $t_{1}, \ldots, t_{n}$ are ground terms. We will sometimes treat a model graph as its corresponding model.

A variable assignment $V$ w.r.t. a Kripke model $M$ is a function that maps each variable to an element of the domain of $M$. The value of $t^{M}[V]$ for a term $t$ is defined as usual.

Given some Kripke model $M=\left\langle D, W, \tau, R_{1}, \ldots, R_{m}, \pi\right\rangle$, some variable assignment $V$, and some world $w \in W$, the satisfaction relation $M, V, w \vDash \psi$ for a formula $\psi$ is defined as follows:

$$
\begin{array}{lll}
M, V, w \vDash p\left(t_{1}, \ldots, t_{n}\right) & \text { iff } & \left(t_{1}^{M}[V], \ldots, t_{n}^{M}[V]\right) \in p^{M, w} ; \\
M, V, w \vDash \square_{i} \varphi & \text { iff } \text { for all } v \in W \text { such that } R_{i}(w, v), M, V, v \vDash \varphi ; \\
M, V, w \vDash \forall x . \varphi & \text { iff } \begin{array}{l}
\text { for all } a \in D,\left(M, V^{\prime}, w \vDash \varphi\right), \\
\\
\end{array} \quad \text { where } V^{\prime}(x)=a \text { and } V^{\prime}(y)=V(y) \text { for } y \neq x ;
\end{array}
$$

and as usual for other cases (treating $\diamond_{i} \varphi$ as $\neg \square_{i} \neg \varphi$, and $\exists x . \varphi$ as $\neg \forall x . \neg \varphi$ ). We say that $M$ satisfies $\varphi$, or $\varphi$ is true in $M$, and write $M \vDash \varphi$, if $M, V, \tau \vDash \varphi$ for every $V$. For a set $\Gamma$ of formulas, we call $M$ a model of $\Gamma$ and write $M \vDash \Gamma$ if $M \vDash \varphi$ for every $\varphi \in \Gamma$.

If as the class of admissible interpretations we take the class of all Kripke models (with no restrictions on the accessibility relations) then we obtain a quantified multimodal logic which has a standard Hilbert-style axiomatization denoted by $K_{m}$. Other normal (multi)modal logics are obtained by adding certain axioms to $K_{m}$. Mostly used axioms are ones that correspond to a certain restriction on the Kripke frame defined by a classical first-order formula using the accessibility relations. For example, the axiom $(D): \square_{i} \varphi \rightarrow \diamond_{i} \varphi$ corresponds to the frame restriction $\forall x \exists y R_{i}(x, y)$.

For a normal modal logic $L$ whose class of admissible interpretations can be characterized by classical first-order formulas of the accessibility relations, we call such formulas $L$-frame restrictions, and call frames with such properties $L$-frames. We call a model $M$ with an $L$-frame an $L$-model. We say that $\varphi$ is $L$ satisfiable if there exists an $L$-model of $\varphi$, i.e. an $L$-model satisfying $\varphi$. A formula $\varphi$ is said to be $L$-valid and called an $L$-tautology if $\varphi$ is true in every $L$-model. For a set $\Gamma$ of formulas, we write $\Gamma \vDash_{L} \varphi$ and call $\varphi$ a logical consequence of $\Gamma$ in $L$ if $\varphi$ is true in every $L$-model of $\Gamma$. 


\subsection{The Multimodal Logic $K D 4 I_{g} 5_{a}$}

Suppose that there are $n$ agents and $m=2^{n}-1$. Let $g$ be an one-to-one function that maps every natural number less than or equal to $m$ to a non-empty subset of $\{1, \ldots, n\}$. Suppose that an index $1 \leq i \leq m$ stands for the group of agents whose indices form the set $g(i)$. To capture belief and common belief of agents, we can extend $K_{m}$ with the following axioms

- (D) : $\square_{i} \varphi \rightarrow \neg \square_{i} \neg \varphi$ (belief is consistent),

- (4) : $\square_{i} \varphi \rightarrow \square_{i} \square_{i} \varphi$ (belief satisfies positive introspection),

- $\left(I_{g}\right): \square_{i} \varphi \rightarrow \square_{j} \varphi$ if $g(i) \supset g(j)$ (if $i$ indicates a supergroup of a group $j$ then every common belief of $i$ is also a common belief of $j$ ).

- ( $\left.5_{a}\right): \neg \square_{i} \varphi \rightarrow \square_{i} \neg \square_{i} \varphi$ if $g(i)$ is a singleton (belief of a single agent satisfies negative introspection).

Thus, for reasoning about belief and common belief, we can use:

$$
K D 4 I_{g} 5_{a}=K_{m}+(D)+(4)+\left(I_{g}\right)+\left(5_{a}\right)
$$

Here we want to catch the most important properties of belief and common belief, and the aim is not to give an exact formulation of belief or common belief. The logic $K D 4 I_{g} 5_{a}$ was introduced in $[20]^{1}$. It is different in the nature from the well-known multimodal logic of common knowledge. It also differs from the modal logic with mutual belief [1].

The given axioms correspond to the following frame restrictions:

Axiom Corresponding Condition

\begin{tabular}{ll}
\hline$(D)$ & $\forall u \exists v R_{i}(u, v)$ \\
$(4)$ & $\forall u, v, w\left(R_{i}(u, v) \wedge R_{i}(v, w) \rightarrow R_{i}(u, w)\right)$ \\
$\left(I_{g}\right)$ & $R_{j} \subseteq R_{i}$ if $g(i) \supset g(j)$ \\
$\left(5_{a}\right)$ & $\forall u, v, w\left(R_{i}(u, v) \wedge R_{i}(u, w) \rightarrow R_{i}(w, v)\right)$ if $g(i)$ is a singleton
\end{tabular}

For further reading on epistemic logics, see, e.g., [10, 28, 7, 1].

\subsection{Modal Logic Programs}

A modality is a (possibly empty) sequence of modal operators. A universal modality is a modality which contains only universal modal operators. We use $\triangle$ to denote a modality and $\square$ to denote a universal modality. Similarly as in classical logic programming, we use a clausal form $\square\left(\varphi \leftarrow \psi_{1}, \ldots, \psi_{n}\right)$ to denote the formula $\forall\left(\square\left(\varphi \vee \neg \psi_{1} \ldots \vee \neg \psi_{n}\right)\right)$. We use $E$ to denote a classical atom and $A$, $B_{1}, \ldots, B_{n}$ to denote formulas of the form $E, \square_{i} E$, or $\diamond_{i} E$.

A program clause is a formula of the form $\square\left(A \leftarrow B_{1}, \ldots, B_{n}\right)$, where $n \geq 0$. 回 is called the modal context, $A$ the head, and $B_{1}, \ldots, B_{n}$ the body of the program clause. An MProlog program is a finite set of program clauses.

\footnotetext{
${ }^{1}$ The propositional version of $K D 4 I_{g} 5_{a}$ is decidable. However, we do not have complexity result for it yet. Hopefully, it will be available in the next version of this paper.
} 
An MProlog goal atom is a formula of the form 回 $E$ or 回 $\diamond_{i} E$. An MProlog goal is a formula written in the clausal form $\leftarrow \alpha_{1}, \ldots, \alpha_{k}$, where each $\alpha_{i}$ is an MProlog goal atom. The empty goal (i.e. the empty clause) is denoted by $\diamond$.

In $K D 4 I_{g} 5_{a}$, if $g(i)$ is a singleton then we have the equivalence $\nabla_{i} \nabla_{i}^{\prime} \varphi \equiv \nabla_{i}^{\prime} \varphi$ for any modal operators $\nabla_{i}$ and $\nabla_{i}^{\prime}$ with the same modal index $i$. For this reason, we adopt some restrictions to simplify the form of MProlog programs and goals in $K D 4 I_{g} 5_{a}$. An MProlog program is called a $K D 4 I_{g} 5_{a}-M P r o l o g$ program if the modal contexts of its program clauses do not contain subsequences of the form $\square_{i} \square_{i}$ if $g(i)$ is a singleton. An MProlog goal is called a $K D 4 I_{g} 5_{a}-$ MProlog goal if each of its goal atoms $\triangle E$ satisfies the condition that $\triangle$ does not contain subsequences of the form $\square_{i} \square_{i}$ or $\square_{i} \diamond_{i}$ if $g(i)$ is a singleton.

Let $P$ be an $K D 4 I_{g} 5_{a}$-MProlog program and $G=\leftarrow \alpha_{1}, \ldots, \alpha_{k}$ be an $K D 4 I_{g} 5_{a}$-MProlog goal. An answer $\theta$ for $P \cup\{G\}$ is a substitution whose domain is the set of all variables of $G$. We say that $\theta$ is a correct answer in $K D 4 I_{g} 5_{a}$ for $P \cup\{G\}$ if $\theta$ is an answer for $P \cup\{G\}$ and $P \vDash_{K D 4 I_{q} 5_{a}} \forall\left(\left(\alpha_{1} \wedge \ldots \wedge \alpha_{k}\right) \theta\right)$.

It is shown in [20] that MProlog has the same expressiveness power as the general Horn fragment in normal modal logics. Moreover, the restrictions adopted for $K D 4 I_{g} 5_{a}$-MProlog do not reduce expressiveness of the language (see [20]).

\section{The Wise Men Puzzle}

Before considering technical details of semantics of $K D 4 I_{g} 5_{a}$-MProlog, we give a formalization of the three wise men puzzle in MProlog. The puzzle is a famous benchmark introduced by McCarthy [17] for AI. It can be stated as follows (cf. [15]). A king wishes to know whether his three advisors (A, B, C) are as wise as they claim to be. Three chairs are lined up, all facing the same direction, with one behind the other. The wise men are instructed to sit down in the order A, B, C. Each of the men can see the backs of the men sitting before them (e.g. C can see A and B). The king informs the wise men that he has three cards, all of which are either black or white, at least one of which is white. He places one card, face up, behind each of the three wise men, explaining that each wise man must determine the color of his own card. Each wise man must announce the color of his own card as soon as he knows what it is. All know that this will happen. The room is silent; then, after a while, wise man A says "My card is white!".

The wise men puzzle has been previously studied in several works (e.g., [17, $15,9,6,2,23,4])$. McCarthy [17] directly used possible worlds to formalize the puzzle. Konolige [15], Nonnengart [23], and Baldoni [4] also used modal logics for the puzzle. Konolige [15] focused on limited reasoning, Nonnengart [23] used semi-functional translation for modal logic programming, and Baldoni [4] used a prefixed tableau system. Both McCarthy [17] and Nonnengart [23] used some feature of mutual belief, but they did not define it purely. Baldoni [4] adopted too strong versions of axioms 4 and 5, which are not suitable for the puzzle. As other approaches for the wise men puzzle, Elgot-Drapkin [9] used step-logics, while Cimatti and Serafini [6], Attardi and Simi [2] studied reasoning in belief- 
contexts. Our formalization of the wise men puzzle given below uses $K D 4 I_{g} 5_{a^{-}}$ MProlog. It is more elegant than the above-mentioned formalizations, as it uses a modal logic with a clear semantics of common belief in a direct way.

As reported in [21], we have designed and implemented a modal logic programming system, also called MProlog. In that system, SLD-resolution calculi for MProlog can be specified according to the theoretical framework given in [22]. An instantiation of that framework for $K D 4 I_{g} 5_{a}$ is presented in the next section. Its implementation (of SLD-resolution) is denoted by ccKD4Ig5a. In that implementation, bel denotes belief and pos denotes possibility, and modalities are represented by lists, e.g. $\square_{i}\langle X\rangle_{j} \diamond_{k} q(a)$ is represented by $[\operatorname{bel}(I), \operatorname{pos}(J, X), \operatorname{pos}(K)]: q(a)$. The implemented calculus requires definitions of predicates singleton_group $/ 1$, subgroup $/ 2$, and union_group $/ 3$. Denote the wise men by $a, b, c$, and the possible groups by $g A B, g A C, g B C, g A B C$, where, e.g., $g A B C=\{a, b, c\}$. Thus, $[\operatorname{bel}(g A B C)]: \varphi$ means that $\varphi$ is a common belief of the group $\{a, b, c\}$. Define the mentioned required predicates in the usual way. The three wise men problem can be formalized by the following program:

:- calculus ccKD4lg5a.

$\%$ If $\mathrm{Y}$ sits behinds $\mathrm{X}$ then $\mathrm{X}$ 's card is white if $\mathrm{Y}$ considers this as possible. [bel $(\mathrm{gABC})]$ : (white $(\mathrm{X})$ :member $(\mathrm{X},[\mathrm{a}, \mathrm{b}, \mathrm{c}])$, member $(\mathrm{Y},[\mathrm{a}, \mathrm{b}, \mathrm{c}]), \mathrm{X} @<\mathrm{Y},[\operatorname{pos}(\mathrm{Y})]$ :white $(\mathrm{X}))$.

$\%$ The following formula is "dual" to the above formula.

$[\operatorname{bel}(\mathrm{gABC})]$ : ([bel $(\mathrm{Y})]$ :black $(\mathrm{X})$ :-

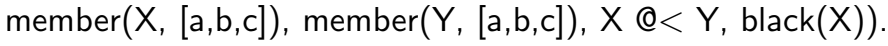

$\%$ At least one of the wise men has a white card.

[bel(gABC)]: (white(a) :- black(b), black(c)).

[bel(gABC)]: (white(b) :- black(c), black(a)).

[bel(gABC)]: (white(c) :- black(a), black(b)).

$/ *$ Each of $\mathrm{B}$ and $\mathrm{C}$ does not know the color of his own card. In particular, each of the men considers that it is possible that his own card is black. */

[bel(gABC), pos(b)]:black(b).

$[$ bel(gABC), pos(c)]:black(c).

The question is whether $A$ believes that his card is white. It is passed to the interpreter as mcall([bel(a)]: white(a)) and solved in less than 1 second $^{2}$ using certain option settings.

The above program uses the syntax of the implemented system. We give below a version using the purely logical formalism of MProlog. For clarity, instead of numeric indices we use $a, b, c, a b, a c, b c, a b c$ with the meaning that $g(a)=\{a\}$, $g(b)=\{b\}, g(c)=\{c\}, \ldots$, and $g(a b c)=\{a, b, c\}$. Let $P_{\text {wise_men }}$ be the following program:

$$
\begin{aligned}
& \varphi_{1}=\square_{a b c}\left(\text { white }(a) \leftarrow \diamond_{b} \text { white }(a)\right) \\
& \varphi_{2}=\square_{a b c}\left(\text { white }(a) \leftarrow \diamond_{c} \text { white }(a)\right)
\end{aligned}
$$

\footnotetext{
${ }^{2}$ on TravelMate $230 \mathrm{X}, 1.7 \mathrm{GHz}-\mathrm{M}$
} 


$$
\begin{aligned}
& \varphi_{3}=\square_{a b c}\left(\text { white }(b) \leftarrow \diamond_{c} \text { white }(b)\right) \\
& \varphi_{4}=\square_{a b c}\left(\square_{b} \operatorname{black}(a) \leftarrow \operatorname{black}(a)\right) \\
& \varphi_{5}=\square_{a b c}\left(\square_{c} \operatorname{black}(a) \leftarrow \operatorname{black}(a)\right) \\
& \varphi_{6}=\square_{a b c}\left(\square_{c} \operatorname{black}(b) \leftarrow \operatorname{black}(b)\right) \\
& \varphi_{7}=\square_{a b c}(\text { white }(a) \leftarrow \operatorname{black}(b), \operatorname{black}(c)) \\
& \varphi_{8}=\square_{a b c}(\text { white }(b) \leftarrow \operatorname{black}(c), \operatorname{black}(a)) \\
& \varphi_{9}=\square_{a b c}(\text { white }(c) \leftarrow \operatorname{black}(a), \operatorname{black}(b)) \\
& \varphi_{10}=\square_{a b c} \diamond_{b} \operatorname{black}(b) \\
& \varphi_{11}=\square_{a b c} \diamond_{c} \text { black }(c)
\end{aligned}
$$

The goal is $\leftarrow \square_{a}$ white $(a)$. We will continue this example in Section 4.5.

For a formalization of the puzzle with $n$ wise men, see [22].

\section{Semantics of $K D 4 I_{g} 5_{a}$-MProlog Programs}

In this section, we present the least model semantics, the fixpoint semantics and an SLD-resolution calculus for $K D 4 I_{g} 5_{a}$-MProlog programs. For abbreviation, from now on we use $L$ to denote KD4I $I_{g} 5_{a}$.

\subsection{Labeled Modal Operators}

When applying the direct consequence operator $T_{L, P}$ for an MProlog program $P$ in $L$, if we obtain an "atom" of the form $\triangle \diamond_{i} E$, then to simplify the task we label the modal operator $\diamond_{i}$. Labeling allows us to address the chosen world(s) in which this particular $E$ must hold. A natural way is to label $\diamond_{i}$ by $E$ to obtain $\langle E\rangle_{i}$. On the other hand, when dealing with SLD-derivation, we cannot change a goal $\leftarrow \diamond_{i}(A \wedge B)$ to $\leftarrow \diamond_{i} A, \diamond_{i} B$. But if we label the operator $\diamond_{i}$, let's say by $X$, then we can safely change $\leftarrow\langle X\rangle_{i}(A \wedge B)$ to $\leftarrow\langle X\rangle_{i} A,\langle X\rangle_{i} B$.

We will use the following notations:

- $T$ : the truth symbol, with the usual semantics ${ }^{3} ;$

- E, F : classical atoms (which may contain variables) or $T$;

- $X, Y, Z$ : variables for classical atoms or $\mathrm{T}$, called atom variables;

- $\langle E\rangle_{i},\langle X\rangle_{i}: \diamond_{i}$ labeled by $E$ or $X$;

- $\nabla: \square_{i}, \diamond_{i},\langle E\rangle_{i}$, or $\langle X\rangle_{i}$, called a modal operator;

$-\triangle:$ a (possibly empty) sequence of modal operators, called a modality;

- 回 : a universal modality;

- $A, B$ : formulas of the form $E$ or $\nabla E$, called simple atoms;

$-\alpha, \beta$ : formulas of the form $\triangle E$, called atoms;

$-\varphi, \psi$ : (labeled) formulas (i.e. formulas that may contain $\langle E\rangle_{i}$ and $\langle X\rangle_{i}$ ).

We use subscripts beside $\nabla$ to indicate modal indexes in the same way as for $\square$ and $\diamond$. To distinguish a number of modal operators we use superscripts of the form $(i)$, e.g. $\square^{(1)}, \square^{(2)}, \nabla^{(i)}, \nabla^{\left(i^{\prime}\right)}$.

\footnotetext{
${ }^{3}$ i.e. it is always true that $M, V, w \vDash \top$
} 
A ground formula is a formula with no variables and no atom variables. A modal operator is said to be ground if it is $\square_{i}, \diamond_{i}$, or $\langle E\rangle_{i}$ with $E$ being $\top$ or a ground classical atom. A ground modality is a modality that contains only ground modal operators. A labeled modal operator is a modal operator of the form $\langle E\rangle_{i}$ or $\langle X\rangle_{i}$.

Denote EdgeLabels $=\left\{\langle E\rangle_{i} \mid E \in \mathcal{B} \cup\{\top\}\right.$ and $\left.1 \leq i \leq m\right\}$, where $\mathcal{B}$ is the Herbrand base (i.e. the set of all ground classical atoms). The semantics of $\langle E\rangle_{i} \in$ EdgeLabels is specified as follows. Let $M=\left\langle D, W, \tau, R_{1}, \ldots, R_{m}, \pi\right\rangle$ be a Kripke model. A $\diamond$-realization function on $M$ is a partial function $\sigma$ : $W \times$ EdgeLabels $\rightarrow W$ such that if $\sigma\left(w,\langle E\rangle_{i}\right)=u$, then $R_{i}(w, u)$ holds and $M, u \vDash E$. Given a $\diamond$-realization function $\sigma$, a world $w \in W$, and a ground formula $\varphi$, the satisfaction relation $M, \sigma, w \vDash \varphi$ is defined in the usual way, except that $M, \sigma, w \vDash\langle E\rangle_{i} \psi$ iff $\sigma\left(w,\langle E\rangle_{i}\right)$ is defined and $M, \sigma, \sigma\left(w,\langle E\rangle_{i}\right) \vDash \psi$. We write $M, \sigma \vDash \varphi$ to denote that $M, \sigma, \tau \vDash \varphi$. For a set $I$ of ground atoms, we write $M, \sigma \vDash I$ to denote that $M, \sigma \vDash \alpha$ for all $\alpha \in I$; we write $M \vDash I$ and call $M$ a model of $I$ if $M, \sigma \vDash I$ for some $\sigma$.

\subsection{Model Generators}

We define that a modality $\nabla_{i_{1}}^{(1)} \ldots \nabla_{i_{k}}^{(k)}$ is in the $L$-normal form if for all $1 \leq j<k$ if $g\left(i_{j}\right)$ is a singleton then $i_{j} \neq i_{j+1}$. (Note that if $g(i)$ is a singleton then $\nabla_{i} \nabla_{i}^{\prime} \varphi \equiv \nabla_{i}^{\prime} \varphi$ is $K D 4 I_{g} 5_{a}$-valid.) A modality is in $L$-normal labeled form if it is in $L$-normal form and does not contain modal operators of the form $\diamond_{i}$ or $\langle\top\rangle_{i}$. An atom is in L-normal (labeled) form if it is of the form $\triangle E$ with $\triangle$ in $L$-normal (labeled) form. An atom is in almost L-normal labeled form if it is of the form $\triangle A$ with $\triangle$ in $L$-normal labeled form.

A model generator is a set of ground atoms not containing $\diamond_{i},\langle\top\rangle_{i}, \top$. An $L$-normal model generator is a model generator consisting of atoms in $L$-normal labeled form.

We will define the standard $L$-model of an $L$-normal model generator $I$ so that it is a least $L$-model of $I$ (where a model $M$ is less than or equal to a model $M^{\prime}$ if for every positive ground formula $\varphi$ without labeled operators, if $M \vDash \varphi$ then $M^{\prime} \vDash \varphi$ ). In the construction we will use the operator $\operatorname{Ext}_{L}$ defined below.

A forward rule is a schema of the form $\alpha \rightarrow \beta$, while a backward rule is a schema of the form $\alpha \leftarrow \beta$. A rule can be accompanied with some conditions specifying when the rule can be applied.

The operator Ext $t_{L}$ is specified by the corresponding forward rules given in Table 1. Given an $L$-normal model generator $I, \operatorname{Ext}_{L}(I)$ is the least extension of $I$ that contains all ground atoms in $L$-normal labeled form that are derivable from some atom of $I$ using the rules specifying $\operatorname{Ext}_{L}$. Note that $\operatorname{Ext}_{L}(I)$ is an $L$-normal model generator if so is $I$.

Denote Serial $_{L}=\left\{\right.$ 回 $\langle T\rangle_{i} \top \mid 1 \leq i \leq m$ and $\square\langle T\rangle_{i}$ is in $L$-normal form $\}$.

Let $I$ be an $L$-normal model generator. The standard $L$-model of $I$ is constructed by building an $L$-model for $\operatorname{Ext}_{L}(I) \cup \operatorname{Serial}_{L}$ according to the semantics of ground labeled modal operators, and formally is defined as follows. Let $W^{\prime}=$ EdgeLabels* (i.e. the set of finite sequences of elements of 


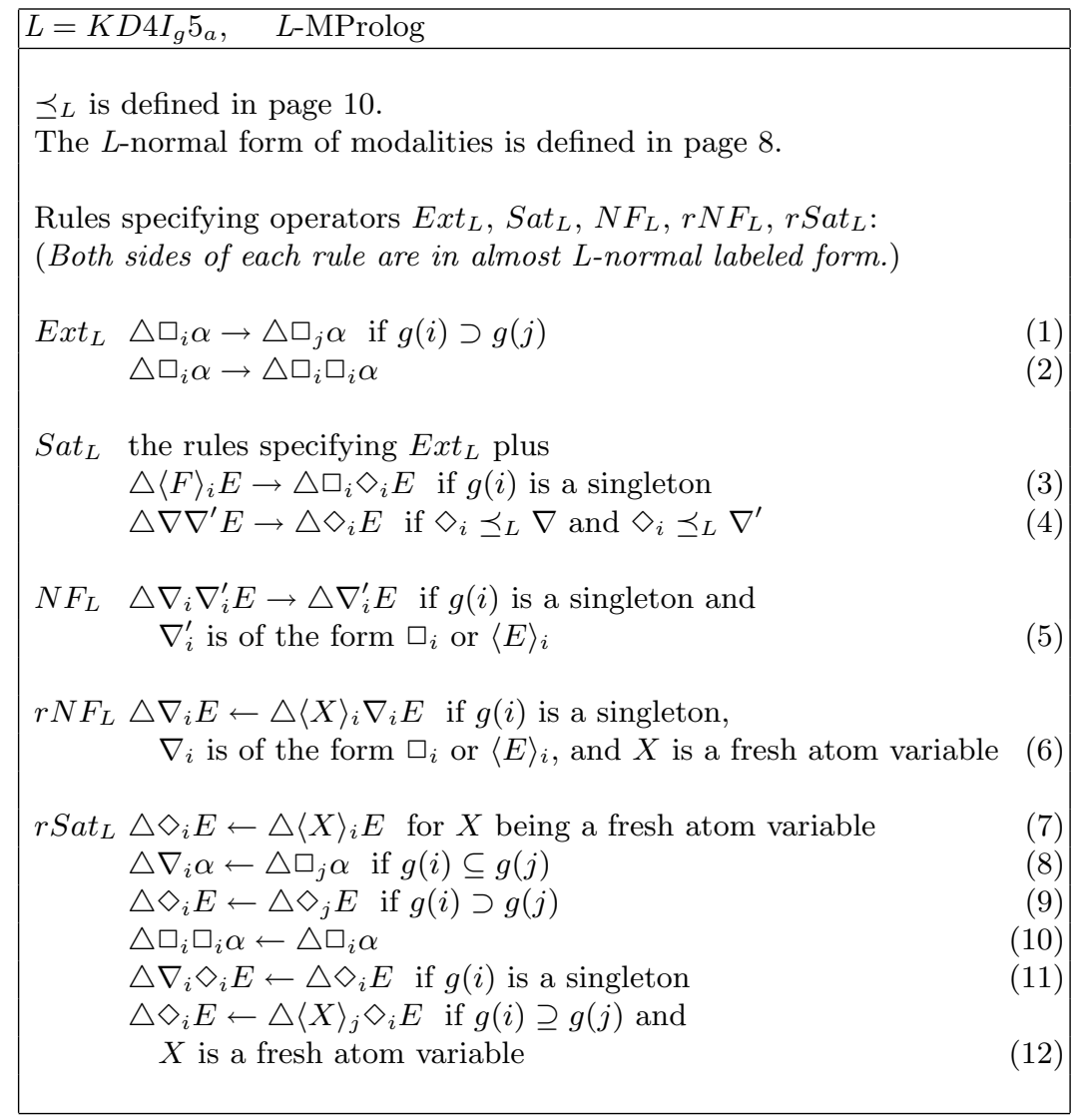

Table 1. A schema for semantics of $K D 4 I_{g} 5_{a}-\mathrm{MProlog}$

$\left\{\langle E\rangle_{i} \mid E \in \mathcal{B} \cup\{\top\}\right.$ and $\left.\left.1 \leq i \leq m\right\}\right), \tau=\epsilon, H(\tau)=\operatorname{Ext}_{L}(I) \cup \operatorname{Serial}_{L}$. Let $R_{i}^{\prime} \subseteq W^{\prime} \times W^{\prime}$ and $H(u)$, for $u \in W^{\prime}, u \neq \tau$, be the least sets such that:

- if $\langle E\rangle_{i} \alpha \in H(w)$, then $R_{i}^{\prime}\left(w, w\langle E\rangle_{i}\right)$ holds and $\{E, \alpha\} \subseteq H\left(w\langle E\rangle_{i}\right)$;

- if $\square_{i} \alpha \in H(w)$ and $R_{i}^{\prime}\left(w, w\langle E\rangle_{i}\right)$ holds, then $\alpha \in H\left(w\langle E\rangle_{i}\right)$.

Let $R_{i}$, for $1 \leq i \leq m$, be the least ${ }^{4}$ extension of $R_{i}^{\prime}$ such that $\left\{R_{i} \mid 1 \leq i \leq m\right\}$ satisfies all the $L$-frame restrictions except seriality (which is cared by Serial $_{L}$ ). Let $W$ be $W^{\prime}$ without worlds not accessible directly nor indirectly from $\tau$ via the accessibility relations $R_{i}$. We call the model graph $\left\langle W, \tau, R_{1}, \ldots, R_{m}, H\right\rangle$ the standard $L$-model graph of $I$, and its corresponding model $M$ the standard L-model of $I .\left\{R_{i}^{\prime} \mid 1 \leq i \leq m\right\}$ is called the skeleton of $M$. By the standard $\diamond$ -

\footnotetext{
${ }^{4}$ the least extension exists due to the assumption that all $L$-frame restrictions not concerning seriality are classical first-order Horn formulas
} 
realization function on $M$ we call the $\diamond$-realization function $\sigma$ defined as follows: if $R_{i}^{\prime}\left(w, w\langle E\rangle_{i}\right)$ holds then $\sigma\left(w,\langle E\rangle_{i}\right)=w\langle E\rangle_{i}$, else $\sigma\left(w,\langle E\rangle_{i}\right)$ is undefined.

It can be shown that the standard $L$-model of an L-normal model generator $I$ is a least $L$-model of $I$.

\subsection{Fixpoint Semantics}

We now consider the direct consequence operator $T_{L, P}$. Given an $L$-normal model generator $I$, how can $T_{L, P}(I)$ be defined? Based on the axioms of $L, I$ is first extended to the $L$-saturation of $I$, denoted by $\operatorname{Sat}_{L}(I)$, which is a set of atoms. Next, L-instances of program clauses of $P$ are applied to the atoms of $\operatorname{Sat}_{L}(I)$. This is done by the operator $T_{0 L, P}$. The set $T_{0}{ }_{, P}\left(\operatorname{Sat}_{L}(I)\right)$ is a model generator but not necessary in $L$-normal form. Finally, the normalization operator $N F_{L}$ converts $T_{0} L, P\left(\operatorname{Sat}_{L}(I)\right)$ to an $L$-normal model generator. $T_{L, P}(I)$ is defined as $N F_{L}\left(T_{0} L, P\left(\operatorname{Sat}_{L}(I)\right)\right)$.

To compare modal operators we define $\preceq_{L}$ to be the least reflexive and transitive relation between modal operators such that $\diamond_{i} \preceq_{L}\langle E\rangle_{i} \preceq_{L} \square_{i}$, $\diamond_{i} \preceq_{L}\langle X\rangle_{i} \preceq_{L} \square_{i}$, and if $g(i) \subseteq g(j)$ then $\square_{i} \preceq_{L} \square_{j}$ and $\diamond_{j} \preceq_{L} \diamond_{i}$.

An atom $\nabla^{(1)} \ldots \nabla^{(n)} \alpha$ is called an $L$-instance of an atom $\nabla^{\left(1^{\prime}\right)} \ldots \nabla^{\left(n^{\prime}\right)} \alpha^{\prime}$ if there exists a substitution $\theta$ such that $\alpha=\alpha^{\prime} \theta$ and $\nabla^{(i)} \preceq_{L} \nabla^{\left(i^{\prime}\right)} \theta$ for all $1 \leq i \leq n$ (treating $\nabla^{\left(i^{\prime}\right)}$ as an expression). For example, if $g(1) \subseteq g(2)$ then $\square_{1} \diamond_{2} E$ is an $L$-instance of $\square_{2}\langle F\rangle_{1} E$.

A modality $\triangle$ is called an $L$-instance of $\triangle^{\prime}$, and we also say that $\triangle^{\prime}$ is equal to or more general in $L$ than $\triangle$ (hereby we define a pre-order between modalities), if $\triangle E$ is an $L$-instance of $\triangle^{\prime} E$ for some ground classical atom $E$.

Let $⿴ 囗$ and $\square^{\prime}$ be universal modalities in $L$-normal form. We say that $⿴ 囗$ is an $L$-context instance of $\mathbf{q}^{\prime}$ if $\square^{\prime} \varphi \rightarrow \square \varphi$ is $L$-valid (for every $\varphi$ ). (It can be shown that the propositional version of the logic $L$ is decidable. So, the problem of checking whether a given universal modality is an $L$-context instance of another one is also decidable.)

Let $⿴$ and $\square^{\prime}$ be universal modalities in $L$-normal form, $\varphi$ and $\varphi^{\prime}$ be program clauses with empty modal context. We say that $\square \varphi$ is an $L$-instance of (a program clause) $\square^{\prime} \varphi^{\prime}$ if 回 is an $L$-context instance of $\square^{\prime}$ and there exists a substitution $\theta$ such that $\varphi=\varphi^{\prime} \theta$.

For example, if $g(1) \subseteq g(2)$ then $\square_{2} \square_{1}$ is an $L$-context instance of $\square_{2}$ and $\square_{2} \square_{1}(p(a) \leftarrow q(a))$ is an $L$-instance of $\square_{2}(p(x) \leftarrow q(x))$.

We now give definitions concerning $\operatorname{Sat}_{L}, T_{0}, P$, and $N F_{L}$.

The saturation operator $S a t_{L}$ is specified by the corresponding forward rules given in Table 1 . Given an $L$-normal model generator $I, \operatorname{Sat}_{L}(I)$ is the least extension of $I$ that contains all ground atoms in almost $L$-normal labeled form that are derivable from some atom in $I$ using the rules specifying $S a t_{L}$. For example, if $g(1)$ is a singleton and $g(2)$ is not, then $\square_{2} \square_{2} \square_{1} \diamond_{1} p(a) \in$ $\operatorname{Sat}_{L}\left(\left\{\square_{2}\langle q(b)\rangle_{1} p(a)\right\}\right)$.

When computing the least fixpoint of a modal logic program, whenever an atom of the form $\triangle \diamond_{i} E$ is introduced, we "fix" the $\diamond$ by replacing the atom by 
$\triangle\langle E\rangle_{i} E$. This leads to the following definition. The forward labeled form of an atom $\alpha$ is the atom $\alpha^{\prime}$ such that if $\alpha$ is of the form $\triangle \diamond_{i} E$ then $\alpha^{\prime}=\triangle\langle E\rangle_{i} E$, else $\alpha^{\prime}=\alpha$. For example, the forward labeled form of $\diamond_{1} s(a)$ is $\langle s(a)\rangle_{1} s(a)$.

Let $P$ be an $L$-MProlog program. The operator $T_{0} L, P$ is defined as follows: for a set $I$ of ground atoms in almost $L$-normal labeled form, $T_{0} L, P(I)$ is the least (w.r.t. $\subseteq$ ) model generator such that if $\left(A \leftarrow B_{1}, \ldots, B_{n}\right)$ is a ground $L$-instance of some program clause of $P$ and $\triangle$ is a maximally general ${ }^{5}$ ground modality in $L$-normal labeled form such that $\triangle$ is an $L$-instance of a and $\triangle B_{i}$ is an $L$-instance of some atom of $I$ (for every $1 \leq i \leq n$ ), then the forward labeled form of $\triangle A$ belongs to $T_{0 L, P}(I)$.

For example, if $g(1) \subseteq g(2)$ and $P$ contains the clause $\square_{2}\left(\diamond_{1} p(x) \leftarrow\right.$ $\left.q(x), r(x), \square_{1} s(x), \diamond_{2} t(x)\right)$ and $I=\left\{\langle q(a)\rangle_{1} q(a), \quad\langle q(a)\rangle_{1} r(a), \square_{2} \square_{2} s(a)\right.$, $\left.\square_{2}\langle t(a)\rangle_{1} t(a)\right\}$, then $\langle q(a)\rangle_{1}\langle p(a)\rangle_{1} p(a) \in T_{0} L, P(I)$.

The normalization operator $N F_{L}$ is specified by the corresponding forward rules given in Table 1. Given a model generator $I, N F_{L}(I)$ is the set of all ground atoms in $L$-normal labeled form that are derivable from some atom of $I$ using the rules specifying $N F_{L}$. For example, if $g(1)$ is a singleton then $N F_{L}\left(\left\{\langle q(a)\rangle_{1}\langle p(a)\rangle_{1} p(a)\right\}\right)=\left\{\langle p(a)\rangle_{1} p(a)\right\}$.

Define $T_{L, P}(I)=N F_{L}\left(T_{0}, P\left(\operatorname{Sat}_{L}(I)\right)\right)$. By definition, the operators $S_{L} t_{L}$, $T_{0} L, P$, and $N F_{L}$ are all increasingly monotonic and compact. Hence the operator $T_{L, P}$ is monotonic and continuous. By the Kleene theorem, it follows that $T_{L, P}$ has the least fixpoint $T_{L, P} \uparrow \omega=\bigcup_{n=0}^{\omega} T_{L, P} \uparrow n$, where $T_{L, P} \uparrow 0=\emptyset$ and $T_{L, P} \uparrow n$ $=T_{L, P}\left(T_{L, P} \uparrow(n-1)\right)$ for $n>0$. Denote the least fixpoint $T_{L, P} \uparrow \omega$ by $I_{L, P}$ and the standard $L$-model of $I_{L, P}$ by $M_{L, P}$.

It can be shown that for an $L$-MProlog program $P, M_{L, P}$ is a least $L$-model of $P$. See also Lemma 1 given in Section 5 .

\subsection{SLD-Resolution}

The main work in developing an SLD-resolution calculus for $L$-MProlog is to specify a reverse analogue of the operator $T_{L, P}$. The operator $T_{L, P}$ is a composition of $S a t_{L}, T_{0} L, P$, and $N F_{L}$. So, we have to investigate reversion of these operators.

A goal is a clause of the form $\leftarrow \alpha_{1}, \ldots, \alpha_{k}$, where each $\alpha_{i}$ is an atom.

The following definition concerns reversion of the operator $T_{0} L, P$.

Let $G=\leftarrow \alpha_{1}, \ldots, \alpha_{i}, \ldots, \alpha_{k}$ be a goal and $\varphi=$ 回 $\left(A \leftarrow B_{1}, \ldots, B_{n}\right)$ a program clause. Then $G^{\prime}$ is derived from $G$ and $\varphi$ in $L$ using mgu $\theta$, and called an $L$-resolvent of $G$ and $\varphi$, if the following conditions hold:

- $\alpha_{i}=\triangle^{\prime} A^{\prime}$, with $\triangle^{\prime}$ in $L$-normal labeled form, is called the selected atom, and $A^{\prime}$ is called the selected head atom;

- $\triangle^{\prime}$ is an $L$-instance of a universal modality $\square^{\prime}$ and $\square^{\prime}\left(A \leftarrow B_{1}, \ldots, B_{n}\right)$ is an $L$-instance of the program clause $\varphi$;

- $\theta$ is an mgu of $A^{\prime}$ and the forward labeled form of $A$;

\footnotetext{
${ }^{5}$ w.r.t. the pre-order between modalities described earlier for $L$
} 
- $G^{\prime}$ is the goal $\leftarrow\left(\alpha_{1}, \ldots, \alpha_{i-1}, \triangle^{\prime} B_{1}, \ldots, \triangle^{\prime} B_{n}, \alpha_{i+1}, \ldots, \alpha_{k}\right) \theta$.

For example, if $g(1) \subseteq g(2)$ then $\leftarrow \square_{1} \diamond_{2} q(x), \square_{1} r(x)$ is an $L$-resolvent of $\leftarrow \square_{1} p(x)$ and $\square_{2}\left(p(x) \leftarrow \diamond_{2} q(x), r(x)\right)$ (here, 回 $=\square_{2}$ and $\triangle^{\prime}=\square^{\prime}=\square_{1}$ ).

As a reverse analogue of the operator $S a t_{L}$, we provide the operator $r S a t_{L}$, which is specified by the corresponding backward rules given in Table 1 . We say that $\beta=r_{S a t}(\alpha)$ using an $r S_{L} t_{L}$ rule $\alpha^{\prime} \leftarrow \beta^{\prime}$ if $\alpha \leftarrow \beta$ is of the form $\alpha^{\prime} \leftarrow \beta^{\prime}$. We write $\beta=r \operatorname{Sat}_{L}(\alpha)$ to denote that " $\beta=r \operatorname{Sat}_{L}(\alpha)$ using some $r \operatorname{Sat}_{L}$ rule".

As a reverse analogue of the operator $N F_{L}$, we provide the operator $r N F_{L}$, which is specified by the corresponding backward rules given in Table 1 . We say that $\beta={ }_{\theta} r N F_{L}(\alpha)$ using an $r N F_{L}$ rule $\alpha^{\prime} \leftarrow \beta^{\prime}$ if $\theta$ is an mgu such that $\alpha \theta \leftarrow \beta$ is of the form $\alpha^{\prime} \leftarrow \beta^{\prime}$. We write $\beta={ }_{\theta} r N F_{L}(\alpha)$ to denote that " $\beta={ }_{\theta} r N F_{L}(\alpha)$ using some $r N F_{L}$ rule". For example, if $g(1)$ is a singleton then we have $\langle Y\rangle_{1}\langle E\rangle_{1} E={ }_{\theta} r N F_{L}\left(\langle X\rangle_{1} E\right)$ with $\theta=\{X / E\}$ and $Y$ being a fresh atom variable.

Let $G=\leftarrow \alpha_{1}, \ldots, \alpha_{i}, \ldots, \alpha_{k}$ be a goal. If $\alpha_{i}^{\prime}=r \operatorname{Sat}_{L}\left(\alpha_{i}\right)$ using an $r \operatorname{Sat}_{L}$ rule $\varphi$, then $G^{\prime}=\leftarrow \alpha_{1}, \ldots, \alpha_{i-1}, \alpha_{i}^{\prime}, \alpha_{i+1}, \ldots, \alpha_{k}$ is derived from $G$ and $\varphi$, and we call $G^{\prime}$ an $(L-)$ resolvent of $G$ and $\varphi$, and $\alpha_{i}$ the selected atom of $G$.

Similarly, $G^{\prime}$ is derived from $G$ and an $r N F_{L}$ rule $\varphi$ using an mgu $\theta$, and called an $\left(L\right.$-) resolvent of $G$ and $\varphi$, if $\alpha_{i}$ is called the selected atom, $\alpha_{i}^{\prime}={ }_{\theta} r N F_{L}\left(\alpha_{i}\right)$ using $\varphi$, and $G^{\prime}=\leftarrow \alpha_{1} \theta, \ldots, \alpha_{i-1} \theta, \alpha_{i}^{\prime}, \alpha_{i+1} \theta, \ldots, \alpha_{k} \theta$.

For example, resolving $\leftarrow \square_{1} \square_{1} p(x)$ with the rule $\triangle \square_{i} \square_{i} \alpha \leftarrow \triangle \square_{i} \alpha$ results in $\leftarrow \square_{1} p(x)$, since $\triangle$ is instantiated to the empty modality, $i$ is instantiated to 1 , and $\alpha$ is instantiated to $p(x)$.

Observe that $r S a t_{L}$ rules and $r N F_{L}$ rules are similar to program clauses and the way of applying them is similar to the way of applying classical program clauses, except that we do not need mgu's for $r S_{t} t_{L}$ rules.

We now define SLD-derivation and SLD-refutation.

Let $P$ be an $L$-MProlog program and $G$ a goal. An $S L D$-derivation from $P \cup\{G\}$ in $L$ consists of a (finite or infinite) sequence $G_{0}=G, G_{1}, \ldots$ of goals, a sequence $\varphi_{1}, \varphi_{2}, \ldots$ of variants of program clauses of $P, r S a t_{L}$ rules, or $r N F_{L}$ rules, and a sequence $\theta_{1}, \theta_{2}, \ldots$ of mgu's such that if $\varphi_{i}$ is a variant of a program clause or an $r N F_{L}$ rule then $G_{i}$ is derived from $G_{i-1}$ and $\varphi_{i}$ in $L$ using $\theta_{i}$, else $\theta_{i}=\varepsilon$ (the empty substitution) and $G_{i}$ is derived from $G_{i-1}$ and (the rSat $_{L}$ rule variant) $\varphi_{i}$. Each $\varphi_{i}$ is called an input clause/rule of the derivation.

We assume standardizing variables apart as usual (see [16]).

An $S L D$-refutation of $P \cup\{G\}$ in $L$ is a finite SLD-derivation from $P \cup\{G\}$ in $L$ with the empty clause as the last goal in the derivation.

Let $P$ be an $L$-MProlog program and $G$ a goal. A computed answer $\theta$ in $L$ of $P \cup\{G\}$ is the substitution obtained by restricting the composition $\theta_{1} \ldots \theta_{n}$ to the variables of $G$, where $\theta_{1}, \ldots, \theta_{n}$ is the sequence of mgu's used in an SLDrefutation of $P \cup\{G\}$ in $L$.

\subsection{Example}

We give here an SLD-refutation of $P_{\text {wise_men }} \cup\left\{\leftarrow \square_{a}\right.$ white $\left.(a)\right\}$ in $K D 4 I_{g} 5_{a}$, where $P_{\text {wise_men }}$ is the $K D 4 I_{g} 5_{a}$-MProlog program given in Section 3 . 


$$
\begin{array}{lrl}
\text { Goals } & \text { Input clauses/rules } & \text { MGUs } \\
\leftarrow \square_{a} \text { white }(a) & & \\
\leftarrow \square_{a} \diamond_{b} \text { white }(a) & \varphi_{1} & \\
\leftarrow \square_{a}\left\langle X_{2}\right\rangle_{b} \text { white }(a) & (7) & \\
\leftarrow \square_{a}\left\langle X_{2}\right\rangle_{b} \diamond_{c} \text { white }(a) & \varphi_{2} & \\
\leftarrow \square_{a}\left\langle X_{2}\right\rangle_{b}\left\langle X_{4}\right\rangle_{c} \text { white }(a) & (7) & \\
\leftarrow \square_{a}\left\langle X_{2}\right\rangle_{b}\left\langle X_{4}\right\rangle_{c} \text { black }(b), \square_{a}\left\langle X_{2}\right\rangle_{b}\left\langle X_{4}\right\rangle_{c} \text { black }(c) & \varphi_{7} \\
\leftarrow \square_{a}\left\langle X_{2}\right\rangle_{b} \text { black }(b), \square_{a}\left\langle X_{2}\right\rangle_{b}\left\langle X_{4}\right\rangle_{c} \text { black }(c) & \varphi_{6} & \\
\leftarrow \square_{a}\langle\text { black }(b)\rangle_{b}\left\langle X_{4}\right\rangle_{c} \text { black }(c) & \varphi_{10} & \left\{X_{2} /\right. \text { black } \\
\diamond & \varphi_{11} & \left\{X_{4} /\right. \text { black }
\end{array}
$$

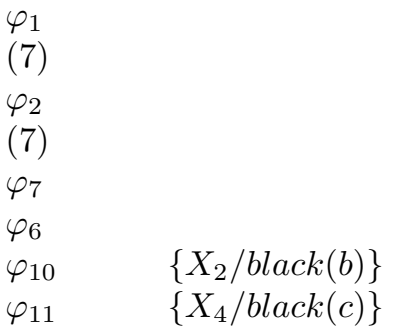

\section{Soundness and Completeness}

In this section, we prove soundness and completeness of the SLD-resolution calculus given for $K D 4 I_{g} 5_{a}$-MProlog, which is stated as follows.

Theorem 1. Let $P$ be an $K D 4 I_{g} 5_{a}-M P r o l o g$ program and $G$ an $K D 4 I_{g} 5_{a^{-}}$ MProlog goal. Then every computed answer in $K D 4 I_{g} 5_{a}$ of $P \cup\{G\}$ is a correct answer in $K D 4 I_{g} 5_{a}$ of $P \cup\{G\}$. Conversely, for every correct answer $\theta$ in $K D 4 I_{g} 5_{a}$ of $P \cup\{G\}$, there exists a computed answer $\gamma$ in $K D 4 I_{g} 5_{a}$ of $P \cup\{G\}$ which is more general than $\theta$ (i.e. $\theta=\gamma \delta$ for some substitution $\delta$ ).

In [22], we presented a general framework for developing fixpoint semantics, the least model semantics, and SLD-resolution calculi for logic programs in multimodal logics, and proved that under certain expected properties of a concrete instantiation of the framework for a specific multimodal logic, the SLD-resolution calculus is sound and complete. The semantics of $K D 4 I_{g} 5_{a}$-MProlog presented in the previous section and summarized in Table 1 is based on and compatible with the framework given in [22].

By the results of [22], to prove soundness and completeness of SLD-resolution of KD4I $5_{a}$-MProlog, we can prove Expected Lemmas 4 - 10 of [22] (w.r.t. the schema given in Table 1). The Expected Lemma 6 is trivial, and the Expected Lemmas 7 - 10, which concern properties of the operators $\operatorname{Sat}_{L}, N F_{L}, r$ Sat $_{L}$, and $r N F_{L}$, can be verified in a straightforward way. The remaining Expected Lemmas 4 and 5 are renumbered respectively as Lemmas 1 and 2 given below.

A model generator $I$ is called an $L$-model generator of $P$ if $T_{L, P}(I) \subseteq I$.

Lemma 1. Let $P$ be an L-MProlog program and $I$ an $L$-model generator of $P$. Then the standard $L$-model of $I$ is an $L$-model of $P$.

Lemma 2. Let $I$ be an L-normal model generator, $M$ the standard L-model of $I$, and $\alpha$ a ground $L$-MProlog goal atom. Suppose that $M \vDash \alpha$. Then $\alpha$ is an $L$-instance of some atom of $\operatorname{Sat}_{L}(I)$.

To prove these lemmas we need Lemmas 3 and 4 given below.

If a modality $\triangle$ is obtainable from $\triangle^{\prime}$ by replacing some (possibly zero) $\nabla_{i}$ by $\square_{i}$ then we call $\triangle$ a $\square$-lifting form of $\triangle^{\prime}$. If $\triangle$ is a $\square$-lifting form of $\triangle^{\prime}$ then 
we call an atom $\triangle \alpha$ a $\square$-lifting form of $\triangle^{\prime} \alpha$. For example, $\square_{1}\langle p(a)\rangle_{1} \square_{2} q(b)$ is a $\square$-lifting form of $\langle X\rangle_{1}\langle p(a)\rangle_{1} \diamond_{2} q(b)$.

Lemma 3. Let $I$ be an L-normal model generator and $M=$ $\left\langle W, \tau, R_{1}, \ldots, R_{m}, H\right\rangle$ the standard L-model graph of I. Let $w=\left\langle E_{1}\right\rangle_{i_{1}} \ldots\left\langle E_{k}\right\rangle_{i_{k}}$ be a world of $M$ and $\triangle=w$ be a modality. Then for $\alpha$ not containing $T$, $\alpha \in H(w)$ iff there exists $a \square$-lifting form $\triangle^{\prime}$ of $\triangle$ such that $\triangle^{\prime} \alpha \in \operatorname{Ext}_{L}(I)$.

This lemma can be easily proved by induction on the length of $\triangle$.

The following lemma is labeled Expected Lemma 2 in [22]. It states that the standard $L$-model of $I$ is really an $L$-model of $I$.

Lemma 4. Let I be an L-normal model generator, $M$ the standard L-model of $I$, and $\sigma$ the standard $\diamond$-realization function on $M$. Then $M$ is an L-model and $M, \sigma \vDash I$.

Proof. By the definition, $M$ is an $L$-model. Let $\left\{R_{i}^{\prime} \mid 1 \leq i \leq m\right\}$ be the skeleton of $M$. We prove by induction on the length of $\alpha$ that for any $w \in W$, if $\alpha \in H(w)$ then $M, \sigma, w \vDash \alpha$. The cases when $\alpha$ is a classical atom or $\alpha=\langle E\rangle_{i} \beta$ are trivial. Consider the remaining case when $\alpha=\square_{i} \beta$. Let $u$ be a world such that $R_{i}(w, u)$ holds. Because $\operatorname{Ext}_{L}(I)$ contains only atoms in $L$-normal form and $\square_{i} \beta \in H(w)$, there does not exist $v$ such that $R_{i}^{\prime}(v, w)$ holds. Consequently, since $R_{i}(w, u)$ holds, there exist worlds $w_{0}=w, w_{1}, \ldots, w_{h-1}, w_{h}=u$ and indices $j_{1}, \ldots, j_{h}$ with $h \geq 1$ such that $R_{j_{1}}^{\prime}\left(w_{0}, w_{1}\right), \ldots, R_{j_{h}}^{\prime}\left(w_{h-1}, w_{h}\right)$, and $g(k) \subseteq g(i)$ for all $k \in\left\{j_{1}, \ldots, j_{h}\right\}$. Since $\square_{i} \beta \in H(w)$, by Lemma 3 , there exists a $\square$-lifting form $\triangle^{\prime}$ of $\triangle=w$ such that $\triangle^{\prime} \square_{i} \beta \in \operatorname{Ext}_{L}(I)$. By the rules specifying $E_{L} t_{L}$, it follows that $\triangle^{\prime} \square_{j_{1}} \ldots \square_{j_{h}} \beta \in \operatorname{Ext}_{L}(I)$. Hence, by Lemma $3, \beta \in H(u)$. By the inductive assumption, $M, \sigma, u \vDash \beta$. Hence $M, \sigma, w \vDash \square_{i} \beta$.

Proof of Lemma 1 Let $M$ be the standard $L$-model of $I$ and $\sigma$ the standard $\diamond$-realization function on $M$. By the definition of $L$-instances of program clauses and the construction of $M$, it is sufficient to prove that for any ground $L$-instance 回 $\left(A \leftarrow B_{1}, \ldots, B_{n}\right)$ of some program clause of $P$, for any $w \in W$ being an $L$-instance of $\square, M, w \vDash\left(A \leftarrow B_{1}, \ldots, B_{n}\right)$. Suppose that $M, w \vDash B_{i}$ for all $1 \leq i \leq n$. We show that $M, w \vDash A$.

Let $\triangle=w=\left\langle E_{1}\right\rangle_{i_{1}} \ldots\left\langle E_{k}\right\rangle_{i_{k}}$. We first show that for any ground simple atom $B$ of the form $E, \square_{i} E$, or $\diamond_{i} E$, if $M, w \vDash B$ then $\triangle B$ is an $L$-instance of some atom from $\operatorname{Sat}_{L}(I)$. Suppose that $M, w \vDash B$. If $k \geq 1$ and $i=i_{k}$ and $g(i)$ is a singleton, then let $v=\left\langle E_{1}\right\rangle_{i_{1}} \ldots\left\langle E_{k-1}\right\rangle_{i_{k-1}}$, else let $v=w$.

If $B=E$, then by Lemma 3 , some $\square$-lifting form of $\triangle B$ belongs to $\operatorname{Ext}_{L}(I)$, and hence $\triangle B$ is an $L$-instance of some atom from $\operatorname{Sat}_{L}(I)$.

Now suppose that $B=\square_{i} E$. Let $u=v\langle\top\rangle_{i}$ and $\triangle^{\prime}=v \square_{i}$. We have $R_{i}(w, u)$, and hence $M, u \vDash E$. By Lemma 3, it follows that some $\square$-lifting form of $\triangle^{\prime} E$ belongs to $\operatorname{Ext}_{L}(I)$. Hence, $\triangle B$ is an $L$-instance of some atom from $\operatorname{Sat}_{L}(I)$.

Next, suppose that $B=\diamond_{i} E$. Consider the case $w \neq v$ (i.e. $i=i_{k}$ and $g(i)$ is a singleton). Since $M, w \vDash B$, there exists $F$ such that $v\langle F\rangle_{i}$ is a world of $M$ and $M, v\langle F\rangle_{i} \vDash E$. Let $\triangle^{\prime}=v\langle F\rangle_{i}$. By Lemma 3, some $\square$-lifting form of $\triangle^{\prime} E$ belongs 
to $\operatorname{Ext}_{L}(I)$. Hence, by the rules (2) and (3) of $S_{a t}, \triangle B$ is an $L$-instance of some atom from $\operatorname{Sat}_{L}(I)$. Now consider the case $w=v$ (i.e. $k=0$ or $i \neq i_{k}$ or $g(i)$ is not a singleton). Since $M, w \vDash \diamond_{i} E$, there exists $u=w\left\langle F_{1}\right\rangle_{j_{1}} \ldots\left\langle F_{h}\right\rangle_{j_{h}}$ such that $M, u \vDash E, h \geq 1$, and $g(l) \subseteq g(i)$ for all $l \in\left\{j_{1}, \ldots, j_{h}\right\}$. By Lemma 3 , some $\square$-lifting form of $w\left\langle F_{1}\right\rangle_{j_{1}} \ldots\left\langle F_{h}\right\rangle_{j_{h}} E$ belongs to $\operatorname{Ext}_{L}(I)$. It follows that some $\square$-lifting form of $\triangle\left\langle F_{1}\right\rangle_{j_{1}} \ldots\left\langle F_{h}\right\rangle_{j_{h}} E$ belongs to $\operatorname{Sat}_{L}(I)$. By the rules of $S_{a} t_{L}$, some $\square$-lifting form of $\triangle \diamond_{i} E$ belongs to $\operatorname{Sat}_{L}(I)$. Hence $\triangle B$ is an $L$-instance of some atom from $\operatorname{Sat}_{L}(I)$.

Since $M, w \vDash B_{i}$ for $1 \leq i \leq n$, it follows that $\triangle B_{i}$ is an $L$-instance of some atom from $\operatorname{Sat}_{L}(I)$. Consequently, $\triangle A$ is an $L$-instance of some atom $\alpha$ from $T_{0, P}\left(\operatorname{Sat}_{L}(I)\right)$. Let $\alpha^{\prime}$ be the $L$-normal form of $\alpha$, i.e. $N F_{L}(\{\alpha\})=\left\{\alpha^{\prime}\right\}$. We have $\alpha^{\prime} \in T_{L, P}(I) \subseteq I$. By Lemma $4, M, \sigma \vDash \alpha^{\prime}$. If $\alpha^{\prime}=\alpha$ then we can derive from $M, \sigma \vDash \alpha^{\prime}$ that $M, w \vDash A$. Suppose that $\alpha^{\prime} \neq \alpha$. Thus, $\alpha$ is of the form $\triangle^{\prime \prime} \nabla_{i} \nabla_{i}^{\prime} E$, where $\triangle^{\prime \prime} \nabla_{i}=\triangle, g(i)$ is a singleton, and $\nabla_{i}^{\prime}$ is $\square_{i}$ or $\langle E\rangle_{i}$. If $\nabla_{i}^{\prime}=\langle E\rangle_{i}$ then $A=\diamond_{i} E$. We have that $\alpha^{\prime}=\triangle^{\prime \prime} \nabla_{i}^{\prime} E$. Since $M, \sigma \vDash \alpha^{\prime}$ and $g(i)$ is a singleton, it follows that $M, \sigma \vDash \triangle^{\prime \prime} \square_{i} A$. Hence $M, w \vDash A$. This completes the proof.

Proof of Lemma 2 Let $\left\langle W, \tau, R_{1}, \ldots, R_{m}, H\right\rangle$ be the standard $L$-model graph of $I$, 回 $=\square_{i_{1}} \ldots \square_{i_{k}}$ be a modality, and $w=\langle T\rangle_{i_{1}} \ldots\langle T\rangle_{i_{k}}$. Suppose that $\alpha$ is of the form $\square$. Since $M \vDash \alpha$, we have $M, w \vDash E$. Hence, by Lemma 3, 回 $E \in \operatorname{Ext}_{L}(I)$, and we also have $\square \in E \operatorname{Sat}_{L}(I)$. Now suppose that $\alpha$ is of the form $\square^{\square} \diamond_{i}$ with the property that if $g(i)$ is a singleton then $i \neq i_{k}$. Since $M \vDash \alpha$, we have $M, w \vDash \diamond_{i} E$. Hence there exists $u=w\left\langle F_{1}\right\rangle_{j_{1}} \ldots\left\langle F_{h}\right\rangle_{j_{h}}$ such that $E \in H(u), h \geq 1$, and $g(l) \subseteq g(i)$ for all $l \in\left\{j_{1}, \ldots, j_{h}\right\}$. By Lemma 3, some $\square$-lifting form of $w\left\langle F_{1}\right\rangle_{j_{1}} \ldots\left\langle F_{h}\right\rangle_{j_{h}} E$ belongs to $\operatorname{Ext}_{L}(I)$. It follows that some $\square$-lifting form of $\square\left\langle F_{1}\right\rangle_{j_{1}} \ldots\left\langle F_{h}\right\rangle_{j_{h}} E$ belongs to $\operatorname{Ext}_{L}(I)$ and $\operatorname{Sat}_{L}(I)$. Hence 四 $\diamond_{i} E$ is an $L$-instance of some atom from $\operatorname{Sat}_{L}(I)$.

We have proved Lemmas 1 and 2, which completes the proof of Theorem 1 .

\section{Conclusions}

Our contributions in this paper are: the schema for semantics of $K D 4 I_{g} 5_{a^{-}}$ MProlog given in Table 1, proofs of the soundness and completeness of SLDresolution for $K D 4 I_{g} 5_{a}-\mathrm{MProlog}$, and a formalization of the wise men puzzle in the purely logical formalism of $K D 4 I_{g} 5_{a}$-MProlog together with its SLDrefutation.

In this text, we recalled a large number of definitions and constructions from [22] (which in turn is an extension of [19]) in order to make the paper selfcontained and understandable. This does not reduce the originality of the abovementioned contributions.

The SLD-refutation given in Section 4.5 for the wise men puzzle does not uses rules or properties involving with axiom $\left(5_{a}\right)$. Consequently, the puzzle can be solved in the logic $K D 4 I_{g}=K_{m}+(D)+(4)+\left(I_{g}\right)$. The choice of $K D 4 I_{g} 5_{a}$ is justified as one of possible multimodal logics of belief and common/mutual belief 
that can be used to formalize the wise men puzzle. Our framework for modal logic programming [22] is applicable for a wide class of multimodal logics and it can be extended for other Kripke model semantics (e.g. with varying domain or non-rigid terms).

This paper considers only one of different aspects of multi-agent systems. In particular, we did not consider temporal dimension, actions, and events. Thus the current version of MProlog is not yet an agent programming language like AgentSpeak(L) [25], 3APL [13], and KARO [18]. To deal with the mentioned aspects, possible solutions are to adopt CTL like the BDI-architecture [26], (concurrent) dynamic logic like the KARO system [18], or discrete linear temporal logic. Extending MProlog with dynamic logic or discrete linear temporal logic is possible, because such logics can be treated as modal logics. However, this is still not sufficient for practical multi-agent systems. There remain a lot of problems to be solved. In our opinion, multi-agent planning deserves for more attention. Also, perhaps we should use rewards and penalties for cooperative and competitive $^{6}$ multi-agent systems to deal with negotiation and cooperation. But in that case, it seems not easy to adopt logics for specification and verification of multi-agent systems.

In summary, this paper is on reasoning about common/mutual belief (which was also considered in the paper [18] on KARO, but neglected in $[26,25,13])$. It shows that the wise men puzzle can be nicely formalized in a multimodal logic of belief using modal logic programming. Our system is goal-driven and we focused on theoretical aspects like soundness and completeness.

\section{References}

1. H. Aldewereld, W. van der Hoek, and J.-J.Ch. Meyer. Rational teams: Logical aspects of multi-agent systems. Fundamenta Informaticae, 63(2-3):159-183, 2004.

2. G. Attardi and M. Simi. Proofs in context. In J. Doyle, E. Sandewall, and P. Torasso, editors, KR'94: Principles of Knowledge Representation and Reasoning, pages 16-26, San Francisco, 1994. Morgan Kaufmann.

3. Ph. Balbiani, L. Fariñas del Cerro, and A. Herzig. Declarative semantics for modal logic programs. In Proceedings of the 1988 International Conference on Fifth Generation Computer Systems, pages 507-514. ICOT, 1988.

4. M. Baldoni. Normal multimodal logics with interaction axioms. In D. Basin, M. D'Agostino, D.M. Gabbay, and L. Viganò, editors, Labelled Deduction, pages 33-57. Kluwer Academic Publishers, 2000.

5. M. Baldoni, L. Giordano, and A. Martelli. A framework for a modal logic programming. In Joint International Conference and Symposium on Logic Programming, pages 52-66. MIT Press, 1996.

6. A. Cimatti and L. Serafini. Multi-agent reasoning with belief contexts: The approach and a case study. In M. Wooldridge and N.R. Jennings, editors, Proceedings of ECAI-94, LNCS 890, pages 71-85. Springer, 1995.

7. N. de Carvalho Ferreira, M. Fisher, and W. van der Hoek. Practical reasoning for uncertain agents. In J.J. Alferes and J.A. Leite, editors, Proceedings of JELIA'2004, volume 3229 of LNCS, pages 82-94. Springer-Verlag, 2004.

\footnotetext{
${ }^{6}$ Environment can be treated as a competitive agent.
} 
8. F. Debart, P. Enjalbert, and M. Lescot. Multimodal logic programming using equational and order-sorted logic. Theoretical Comp. Science, 105:141-166, 1992.

9. J.J. Elgot-Drapkin. Step-logic and the three-wise-men problem. In $A A A I$, pages 412-417, 1991

10. R. Fagin, J.Y. Halpern, Y. Moses, and M.Y. Vardi. Reasoning About Knowledge. MIT Press, 1995.

11. L. Fariñas del Cerro. Molog: A system that extends Prolog with modal logic. New Generation Computing, 4:35-50, 1986.

12. M. Fisher and R. Owens. An introduction to executable modal and temporal logics. In M. Fisher and R. Owens, editors, Executable Modal and Temporal Logics, IJCAI'93 workshop, pages 1-20. Springer, 1995.

13. K.V. Hindriks, F.S. De Boer, W. van der Hoek, and J.-J.Ch. Meyer. Agent programming in 3APL. Autonomous Agents and Multi-Agent Systems, 2(4):357-401, 1999.

14. M. Kacprzak, A. Lomuscio, and W. Penczek. Bounded versus unbounded model checking for interpreted systems (invited talk at FAAMAS'03). In B. Dunin-Keplicz and R. Verbrugge, editors, Proceedings of FAAMAS'03, pages 5-20, 2003.

15. K. Konolige. Belief and incompleteness. Technical Report 319, SRI Inter., 1984

16. J.W. Lloyd. Foundations of Logic Programming, 2nd Ed. Springer-Verlag, 1987.

17. J. McCarthy. First order theories of individual concepts and propositions. Machine Intelligence, 9:120-147, 1979.

18. J.-J.Ch. Meyer, F.S. de Boer, R.M. van Eijk, K.V. Hindriks, and W. van der Hoek. On programming KARO agents. Logic Journal of the IGPL, 9(2), 2001.

19. L.A. Nguyen. A fixpoint semantics and an SLD-resolution calculus for modal logic programs. Fundamenta Informaticae, 55(1):63-100, 2003.

20. L.A. Nguyen. Multimodal logic programming and its applications to modal deductive databases. Manuscript (served as a technical report), available on Internet at http://www . mimuw.edu.pl/〜nguyen/papers.html, 2003.

21. L.A. Nguyen. The modal logic programming system MProlog. In J.J. Alferes and J.A. Leite, editors, Proceedings of JELIA 2004, LNCS 3229, pages 266-278. Springer, 2004.

22. L.A. Nguyen. The modal logic programming system MProlog: Theory, design, and implementation. Available at http://www.mimuw.edu.pl/ nguyen/mprolog, 2005.

23. A. Nonnengart. How to use modalities and sorts in Prolog. In C. MacNish, D. Pearce, and L.M. Pereira, editors, Proceedings of JELIA'94, LNCS 838, pages 365-378. Springer, 1994.

24. M.A. Orgun and W. Ma. An overview of temporal and modal logic programming. In D.M. Gabbay and H.J. Ohlbach, editors, Proc. First Int. Conf. on Temporal Logic - LNAI 827, pages 445-479. Springer-Verlag, 1994.

25. A.S. Rao. AgentSpeak(L): BDI agents speak out in a logical computable language. In Proceedings of the 7th European Workshop MAAMAW, volume 1038 of LNCS, pages 42-55. Springer, 1996.

26. A.S. Rao and M.P. Georgeff. Modeling rational agents within a BDI-architecture. In $K R$, pages $473-484,1991$.

27. R.A. Schmidt and D. Tishkovsky. Multi-agent logic of dynamic belief and knowledge. In S. Flesca, S. Greco, N. Leone, and G. Ianni, editors, Proceedings of JELIA'2002, volume 2424 of LNAI, pages 38-49. Springer, 2002.

28. W. van der Hoek and J.-J. Meyer. Modalities for reasoning about knowledge and uncertainties. In P. Doherty, editor, Partiality, Modality, and Nonmonotonicity. CSLI Publications, 1996. 